\title{
$A b$ initio potential energy curve for the ground state of beryllium dimer
}

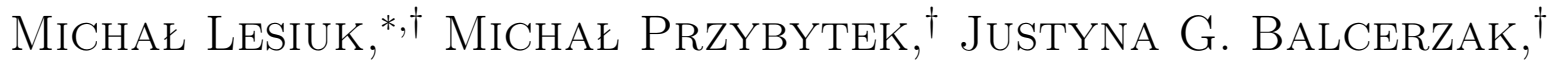 \\ Monika Musiat,$^{\ddagger}$ And Robert Moszynski ${ }^{\dagger}$ \\ $\dagger$ Faculty of Chemistry, University of Warsaw, Pasteura 1, 02-093 Warsaw, Poland \\ $\ddagger$ Institute of Chemistry, University of Silesia, Szkolna 9, 40-006 Katowice, Poland \\ E-mail: e-mail:lesiuk@tiger.chem.uw.edu.pl
}

\begin{abstract}
This work concerns ab initio calculations of the complete potential energy curve and spectroscopic constants for the ground state $X^{1} \Sigma_{g}^{+}$of the beryllium dimer, $\mathrm{Be}_{2}$. High accuracy and reliability of the results is one of the primary goals of the paper. To this end we apply large basis sets of Slater-type orbitals combined with highlevel electronic structure methods including triple and quadruple excitations. The effects of the relativity are also fully accounted for in the theoretical description. For the first time the leading-order quantum electrodynamics effects are fully incorporated for a many-electron molecule. Influence of the finite nuclear mass corrections (post-Born-Oppenheimer effects) turns out to be completely negligible for this system. The predicted well-depth $\left(D_{e}=934.5 \pm 2.5 \mathrm{~cm}^{-1}\right)$ and the dissociation energy $\left(D_{0}=808.0 \mathrm{~cm}^{-1}\right)$ are in a very good agreement with the most recent experimental data. We confirm the existence of the weakly bound twelfth vibrational level [Patkowski et al., Science 326, 1382 (2009)] and predict that it lies just about $0.5 \mathrm{~cm}^{-1}$ below the onset of the continuum.
\end{abstract}




\section{Introduction}

In the past decades beryllium dimer has been the subject of many studies, both experimental and theoretical. The first calculations predicted the interaction between two closed-shell beryllium atoms to be purely repulsive, $\underline{\underline{1}}$ even when the electron correlation effects were partially included. $\stackrel{2}{2}$ However, more sophisticated quantum chemistry methods became available in the late 70' and early 80' allowing to re-evaluate the scientific consensus about the nature of the bonding in the beryllium dimer. $\cdot^{\underline{3}}-\underline{5}$ It was predicted that this molecule is bound, albeit weakly, with some similarities to the noble gas dimers.

Further improvements in the theoretical description of the beryllium dimer were presented by Liu and McLean, $\underline{\underline{6}}$ and somewhat later by Harrison and Handy. $\underline{\underline{7}}$ Both studies reported that the single and double excitations with respect to the single reference wavefunction are not sufficient to describe the bonding correctly. Inclusion of triple and quadruple excitations (either by means of full CI or multireference methods ${ }^{8-10}$ ) is necessary to obtain more quantitative results. This allowed to revise the bonding energy up to several hundreds of $\mathrm{cm}^{-1}$. Moreover, it was shown that the pathological behaviour of this system is largely due to the near-degeneracy of the $2 s$ and $2 p$ energy levels of the beryllium atom. These conclusions have been confirmed by several other authors. $\underline{11}-\underline{18}$

The fact that the beryllium dimer is an apparently simple yet challenging system has made it a frequent subject of state-of-the-art computational studies. At present the consensus is that the binding energy of the beryllium dimer is in the range $920-940 \mathrm{~cm}^{-1}$ and the bond length is approximately $2.44 \AA$. The reported values differ depending on the employed level of theory but it appears that the most reliable theoretical results to date were given by Martin $\underline{19}\left(944 \pm 25 \mathrm{~cm}^{-1}\right)$, Røggen and $\operatorname{Veseth}^{20}\left(945 \pm 15 \mathrm{~cm}^{-1}\right)$, Patkowski et al. $\underline{21}$ $\left(938 \pm 15 \mathrm{~cm}^{-1}\right)$, Koput $^{22}\left(935 \pm 10 \mathrm{~cm}^{-1}\right)$, and the present authors ${ }^{23}\left(929 \pm 1.9 \mathrm{~cm}^{-1}\right)$.

Other notable papers are Refs. [24-30] and a more detailed older bibliography is found in Refs. [20, 21]. Semiempirical (or morphed) potentials have also been constructed for this system. $\underline{31}, \underline{32}$ 
Experimental studies of the beryllium dimer ground state also have a long history. The first experimental works of Bondybey et al. $\underline{\underline{33}}-\underline{35}$ were conducted in the middle $80^{\prime}$ and only a few vibrational levels were observed. These incomplete data and a lacking theoretical model led to a considerably underestimated value for the well-depth, $790 \pm 30 \mathrm{~cm}^{-1}$. This prediction was later revised by Spirko $\underline{\underline{31}}$ who combined the experimental results of Bondybey et al. $\underline{33-35}$ with portions of theoretical potentials and recommended a new value of $923 \mathrm{~cm}^{-1}$.

A refined experiment was performed in 2009 by Merritt et al. $\underline{\underline{36}}$ who reported $929.7 \pm 2$ $\mathrm{cm}^{-1}$ for the well-depth. In addition, eleven vibrational levels were characterised. $\underline{\underline{37}}$ However, to extract the potential parameters (well-depth, equilibrium distance, etc.) from the experimental results, Merritt et al. $\underline{\underline{36}}$ employed a relatively simple Morse-like potential. It vanishes too fast (i.e. exponentially) at large internuclear distances. This deficiency was corrected by Patkowski et al. $\underline{\underline{38}}$ who calculated a theoretical potential energy curve with the correct $R^{-6}$ long-range behaviour, where $R$ is the internuclear distance. This potential was not accurate enough to reproduce the experimental results with the spectroscopic accuracy, but by a simple morphing of the potential the accuracy was greatly improved. By introducing two empirical parameters they reproduced the experimental vibrational levels to within $1.0 \mathrm{~cm}^{-1}$, and with five parameters the error was further reduced to about $0.1 \mathrm{~cm}^{-1}$.

Even more interestingly, the morphed potential of Patkowski et al. $\underline{\underline{38}}$ supported an additional (i.e. twelfth) vibrational level. This level was not originally reported in the experimental paper of Merritt et al. $\underline{\underline{36}}$ and its existence came as a surprise. Several subsequent

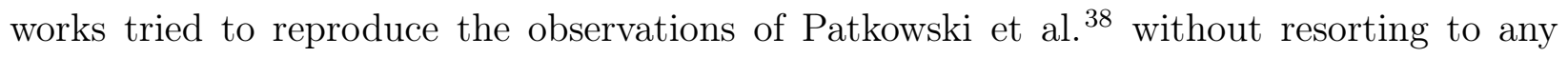
empirical adjustments. ${ }^{22}$ In parallel, refined direct-potential-fit analyses provided improved (albeit purely empirical) potentials, $\underline{32}$ supporting the findings of Ref. [38].

In this paper we expand upon our previous work $\frac{23}{}$ where the interaction energy of the beryllium dimer at the minimum of the potential energy curve has been determined with help of the Slater-type orbitals $\underline{39}, \underline{40}$ by using the newly developed programs. $\underline{41}^{-43}$ We largely extend the results reported previously $\underline{\underline{23}}$ and calculate the full potential energy curve (PEC) 
including corrections due to the adiabatic, relativistic, and quantum electrodynamics effects. Next, we generate analytic fits of the interaction potentials and solve the nuclear Schrödinger equation to obtain the vibrational energy terms. Finally, an extensive comparison with the existing theoretical and experimental data is given.

Atomic units are used throughout the paper unless explicitly stated otherwise. We adopt the following conversion factors and fundamental constants: $1 a_{0}=0.529177 \AA$ (Bohr radius), $1 \mathrm{u}=1822.888$ (unified atomic mass unit), $1 \mathrm{H}=219474.63 \mathrm{~cm}^{-1}$ (Hartree), $\alpha=$ 1/137.035 999 (the fine structure constant). These values are in line with the recent CODATA recommendations. $\underline{\underline{44}}$ We assume that the mass of the only stable isotope of beryllium $\left({ }^{9} \mathrm{Be}\right)$ is $m(\mathrm{Be})=9.012183 \mathrm{u}$ which is the latest experimental value. ${ }^{45}$ All data presented in this paper refer to the ${ }^{9}$ Be isotope. We also adopt a convention that the interaction energy is positive whenever the underlying interaction is attractive.

\section{Ab initio calculations}

\subsection{Basis sets}

In this work we use basis sets composed of the canonical Slater-type orbitals 39,40

$$
\chi_{l m}(\mathbf{r} ; \zeta)=\frac{(2 \zeta)^{n+1 / 2}}{\sqrt{(2 n) !}} r^{l} e^{-\zeta r} Y_{l m}(\theta, \phi)
$$

where $\zeta>0$ is a nonlinear parameter to be optimised, and $Y_{l m}$ are spherical harmonics in the Condon-Shortley phase convention. In our previous paper 23 the optimisation of the STOs basis sets has been described in detail. It the present work we employ slightly modified procedures, so let us describe the most important differences. First, instead of the conventional well-tempering of the nonlinear parameters for a given angular momentum $\left(\zeta_{l k}\right)$ we employ 
Table 1: Composition of the STO basis sets for the beryllium atom used in this work.

\begin{tabular}{l|lll}
\hline$l$ & valence & core & diffuse \\
\hline 2 & $7 s 2 p 1 d$ & $1 s 1 p$ & $2 s 2 p 1 d$ \\
3 & $8 s 3 p 2 d 1 f$ & $2 s 2 p 1 d$ & $2 s 2 p 2 d 1 f$ \\
4 & $9 s 4 p 3 d 2 f 1 g$ & $2 s 3 p 2 d 1 f$ & $2 s 2 p 2 d 2 f 1 g$ \\
5 & $9 s 5 p 4 d 3 f 2 g 1 h$ & $3 s 4 p 3 d 2 f 1 g$ & $2 s 2 p 2 d 2 f 2 g 1 h$ \\
6 & $9 s 6 p 5 d 4 f 3 g 2 h 1 i$ & $3 s 5 p 4 d 3 f 2 g 1 h$ & $2 s 2 p 2 d 2 f 2 g 2 h 1 i$ \\
\hline
\end{tabular}

a more flexible formula

$$
\zeta_{l k}=\alpha_{l} \beta_{l}^{k+\gamma_{l} k^{2}} \quad \text { with } \quad k=0,1,2, \ldots
$$

where $\alpha_{l}, \beta_{l}$, and $\gamma_{l}$ are free parameters to be optimised. For a brief discussion of advantages of this expansion see Ref. [46].

Similarly as in the previous works we divide the basis sets into the core and valence components and employ the correlation-consistency principle $\underline{\underline{47}}$ to determine the final composition of both parts. However, in contrast to Ref. [23] an additional set of diffuse functions is added to each basis. Therefore, all basis sets used here are doubly augmented. The low-exponent functions are especially beneficial for larger internuclear distances. The final composition of all basis sets is given in Table 1. Other details can be obtained from the authors upon request. For brevity, the valence-only basis sets are denoted shortly wtcc-l whilst the core-valence basis sets are abbreviated tc-wtcc- $l$. In both cases, $l$ is the highest angular momentum present in the basis set and the double augmentation is denoted with the prefix da-, e.g. da-wtcc-l.

Special basis sets are used further in the paper for the calculations of the relativistic and QED effects. In this case we modify the original da-tc-wtcc-l basis sets by replacing all $s$-type functions by a common set of twelve $1 s$ orbitals. This set has been obtained by minimising the Hartree-Fock energy of the beryllium atom. Detailed compositions of the STO basis sets used in this work (exponents and quantum numbers) are given in the supplementary material. 
Table 2: The total nonrelativistic $\left(E_{\text {total }}\right)$ and correlation energies $\left(E_{\mathrm{c}}\right)$ of the beryllium atom calculated at the FCI level of theory (see the main text for details). The limit of the Hartree-Fock energy is -14.573023 a.u. (all digits given are accurate).

\begin{tabular}{lrrc}
\hline$l$ & $N$ & $E_{\mathrm{c}} / \mathrm{mH}$ & $E_{\text {total }}$ \\
\hline 2 & 31 & -85.976 & -14.658998 \\
3 & 67 & -91.479 & -14.664502 \\
4 & 124 & -92.994 & -14.666017 \\
5 & 204 & -93.608 & -14.666631 \\
6 & 316 & -93.902 & -14.666925 \\
\hline CBS & $\infty$ & -94.429 & -14.667452 \\
\hline Ref. [49] & & -94.333 & -14.667356 \\
\hline
\end{tabular}

In Table. 2 we present results of the FCI calculations for the beryllium atom in the da-tc-wtcc-l basis sets. The Hartree-Fock (HF) limit is reached already with the basis set $l=5$ and we did not attempt to extrapolate the HF results. The correlation energies are extrapolated with the help of the following formula $\underline{48}$

$$
E=a+b(l+1)^{-3}
$$

where $l$ is the highest angular momentum present in the basis, and the parameters $a, b$ are obtained by least-squares fitting. The best quality of the results for the beryllium atom is obtained by extrapolation from the $l=4,5,6$ basis sets. Two-point extrapolation from $l=5,6$ also yields good results and we found it useful in estimating the extrapolation errors. The formula (3) with $l=4,5,6$ will be used in all subsequent molecular calculations for the extrapolation of the correlation energies. In Table 2 we show that the extrapolated results for the beryllium atom differ from the reference values of Pachucki and Komasa $\stackrel{49}{ }$ by less than $100 \mu \mathrm{H}$. Moreover, the extrapolation reduces the error of the largest basis set by a factor of five. 


\subsection{Four-electron (valence) contribution}

Within the current computational capacities the full CI (FCI) method cannot be used for eight-electron systems with any reasonable basis set. Therefore, in the present work we rely on a composite scheme where the total interaction energy is divided into a set of welldefined components of different magnitudes. The largest components are calculated most accurately, i.e. employing larger basis sets or more reliable electronic structure methods. Smaller contributions are treated at a more approximate level of theory or even completely neglected.

It is well-known that the dominant contribution to the interaction energy of the beryllium dimer comes from the outer valence electrons. In fact, by freezing the $1 s$ core orbitals of both atoms one can still recover approximately $90 \%$ of the total interaction energy. Unfortunately, calculation of the valence four-electron contribution is challenging due to the aforementioned $2 s-2 p$ near-degeneracy of the energy levels of the beryllium atom. This leads to a significant multireference character of the dimer. As a result, $\operatorname{CCSD}(\mathrm{T})$ (or even CCSDT) method should not be used in an accurate calculation of the valence four-electron contribution to the interaction energy. To get a quantitative answer one has to use either the FCI method or some multireference $\mathrm{CI} / \mathrm{CC}$ variant. In the present paper we choose the former option, mostly because of its black-box character and no arbitrariness, e.g. in the selection of the active orbital space.

Valence four-electron FCI interaction energies were calculated with the basis sets dawtcc- $l, l=2-6$. This was accomplished by using the FCI program HeCTOR $\underline{50}$ written by one of us (MP). Canonical Hartree-Fock orbitals generated by external programs were used as a starting point for the FCI iterations. All FCI computations were performed utilising the $D_{2 h}$ Abelian point group symmetry. The largest basis set leads to a FCI matrix of dimension over one billion $\left(10^{9}\right)$. Basis set superposition error is eliminated by applying the counterpoise correction. $\underline{\underline{51}}$ In Table 3 we present exemplary results of the valence FCI calculations. To provide a broader picture we list these data for two interelectronic distances, 
Table 3: Four-electron valence calculations for the beryllium dimer; $l$ is the largest angular momentum present in the basis set; $N_{b}$ is the number of basis set functions, $E_{\mathrm{int}}^{\mathrm{HF}}$ and $E_{\mathrm{int}}^{\mathrm{FCI}}$ are interaction energies calculated at the Hartree-Fock and FCI levels of theory, respectively. Results are given for two internuclear distances, $R$. The interaction energies are given in $\mathrm{cm}^{-1}$ and the internuclear distances in bohr.

\begin{tabular}{crcccc}
\hline$l$ & $N_{b}$ & \multicolumn{2}{c}{$R=4.75$} & \multicolumn{2}{c}{$R=8.00$} \\
\hline & & $E_{\text {int }}^{\mathrm{HF}}$ & $E_{\text {int }}^{\mathrm{FCI}}$ & $E_{\text {int }}^{\mathrm{HF}}$ & $E_{\text {int }}^{\mathrm{FCI}}$ \\
\hline 2 & 54 & -2367.5 & 270.3 & -124.5 & 116.2 \\
3 & 110 & -2324.3 & 692.2 & -123.5 & 155.0 \\
4 & 192 & -2320.6 & 804.8 & -123.3 & 167.7 \\
5 & 302 & -2320.5 & 831.7 & -123.2 & 171.1 \\
6 & 448 & -2320.4 & 842.7 & -123.2 & 172.0 \\
\hline$\infty$ & $\infty$ & $-2320.4 \pm 0.1$ & $865.5 \pm 2.0$ & $-123.2 \pm 0.1$ & $174.8 \pm 1.2$ \\
\hline
\end{tabular}

$R=4.75$ located near the minimum of PEC and $R=8.0$, already close to the asymptotic van der Waals region.

To reach the basis set limit of the calculated quantities and estimate the corresponding errors we rely on the CBS extrapolations. The only exception is the Hartree-Fock (HF) energy. As one can see from Table 3 the HF contribution to the interaction energy is converged to better than $0.1 \mathrm{~cm}^{-1}$ already in the basis set $l=6$. Therefore, we simply take the value obtained with $l=6$ as the HF limit. The error of this approximation is negligible in the present context. A more complicated situation is found for the contributions coming from the correlation energy. To extrapolate them we employ the same formula which has been demonstrated to give reliable results for an isolated atom, Eq. (3). Overall, we find that the formula (3) fits the raw data points very well. The extrapolated values of the interaction energy (FCI level of theory) are also listed in Table 3. The errors are estimated as half of the difference between the extrapolated results from the basis sets $l=4,5,6$ and $l=5,6$.

Let us also illustrate how important the post-CCSD $(\mathrm{T})$ effects are in the calculation of the valence contributions to the interaction energy. For example, the interaction energy calculated with the frozen-core $\operatorname{CCSD}(\mathrm{T})$ method $\underline{52}$ and the $l=6$ basis set is $623.9 \mathrm{~cm}^{-1}$ for the $R=4.75$ and $59.6 \mathrm{~cm}^{-1}$ for $R=8.0$. Comparison with the values calculated at the 
FCI level of theory (cf. Table 3) shows that $\operatorname{CCSD}(\mathrm{T})$ recovers only approx. $75 \%$ of the total valence interaction energy for $R=4.75$ and $90 \%$ for $R=8.0$. These deviations cannot be attributed to the basis set incompleteness error since a very similar picture is obtained from the CBS-extrapolated data. Therefore, the $\operatorname{CCSD}(\mathrm{T})$ method alone is not a reasonable level of theory for the calculation of the valence contribution to the interaction energy of the beryllium dimer.

Table 4: Core-core and core-valence contributions $\left(E_{\mathrm{int}, \mathrm{X}}^{\mathrm{X}}\right)$ to the interaction energy of the beryllium dimer calculated at various levels of theory (X) in the da-tc-wtcc-l basis sets (see the main text for precise definitions of all quantities); $N_{b}$ is the number of basis set functions. The interaction energies are given in $\mathrm{cm}^{-1}$ and the internuclear distances in bohr.

\begin{tabular}{rrcccccc}
\hline$l$ & $N_{b}$ & \multicolumn{3}{c}{$R=4.75$} & & \multicolumn{3}{c}{$R=8.00$} \\
\hline & & $E_{\text {int,core }}^{\mathrm{CCSD}(\mathrm{T})}$ & $\Delta E_{\text {int,core }}^{\mathrm{T}}$ & $\Delta E_{\text {int,core }}^{(\mathrm{Q})}$ & $E_{\text {int,core }}^{\mathrm{CCSD}(\mathrm{T})}$ & $\Delta E_{\text {int,core }}^{\mathrm{T}}$ & $\Delta E_{\text {int,core }}^{(\mathrm{Q})}$ \\
\hline 2 & 54 & 26.1 & -7.4 & 1.0 & +0.2 & -0.8 & +0.4 \\
3 & 110 & 50.8 & -4.6 & 0.6 & -1.1 & -0.8 & +0.5 \\
4 & 192 & 54.1 & -3.3 & - & -1.6 & - & - \\
5 & 302 & 54.8 & - & - & -1.7 & - & - \\
\hline$\infty$ & $\infty$ & $55.6 \pm 0.8$ & $-2.3 \pm 0.5$ & $0.4 \pm 0.2$ & $-1.9 \pm 0.2$ & $-0.8 \pm 0.4$ & $+0.6 \pm 0.3$ \\
\hline
\end{tabular}

\subsection{Core-core and core-valence contributions}

Let us now consider the contribution to the total interaction energy coming from the corecore and core-valence (i.e. inner-shell) correlations, $E_{\text {int(core) }}$. It is defined as the difference between the exact nonrelativistic Born-Oppenheimer (BO) interaction energy and the exact four-electron valence contribution. Fortunately, calculation of this contribution is simpler in some respects than of the valence effects. The largest contribution to $E_{\text {int(core) }}$ can be obtained with the $\operatorname{CCSD}(\mathrm{T})$ method, $E_{\text {int(core) }}^{\mathrm{CCSD}(\mathrm{T})}$. The post-CCSD(T) contributions to $E_{\text {int(core) }}$ constitute only a few percents of the exact value - a stark contrast to the previous case of $E_{\text {int }}^{\mathrm{FCI}}$.

In Table 4 we present the inner-shell contributions to the interaction energy $\left(E_{\mathrm{int}, \mathrm{X} \text { core }}^{\mathrm{X}}\right)$ calculated at several different levels of theory, X. In this work we consider $\mathrm{X}=\mathrm{CCSD}(\mathrm{T})$, 
CCSDT or CCSDT(Q). For convenience, let us also define some relative quantities

$$
\begin{aligned}
& \Delta E_{\text {int }, \text { core }}^{\mathrm{T}}=E_{\text {int(core })}^{\mathrm{CCSDT}}-E_{\text {int(core })}^{\mathrm{CCSD}(\mathrm{T})}, \\
& \Delta E_{\text {int }, \text { core }}^{\mathrm{(Q)}}=E_{\text {int(core })}^{\mathrm{CCSDT}(\mathrm{Q})}-E_{\text {int(core })}^{\mathrm{CCSDT}},
\end{aligned}
$$

Calculation of the above post-CCSD $(\mathrm{T})$ corrections is computationally very intensive. For example, single-point CCSDT calculations for the dimer in the $l=4$ basis take about a month with our computational resources. The cost of the CCSDT(Q) method is even higher which effectively prohibits the use of basis sets larger than $l=2,3$. In the case of the CCSDT method we managed to perform calculations up to $l=4$ only for several points on the PEC, namely $R=4.0-5.5$ a.u. This is the region where the interaction energy is the largest and the inner-shell corrections are the most important on the relative scale. In fact, for $R=4.75$ the inner-shell contributions stand for about $8 \%$ of the total interaction energy in the BO approximation (cf. Table 3). For $R=8.0$ this ratio drops to less than $2 \%$.

Extrapolations of the $\operatorname{CCSD}(\mathrm{T})$ results to the CBS limit are performed with the help of the formula (3) with $l=4-5$. The errors are estimated as differences between the respective values calculated with the largest basis set and the extrapolated limit. The same technique is used for the CCSDT method where the results from $l=2,3,4$ basis sets are available. In this case the error is estimated as $20 \%$ of the extrapolated value. For the remaining data we extrapolate the results by using the $l=2,3$ basis sets. Clearly, this approach is not as reliable as the other estimates employed in this work and thus we assign an uncertainty of $50 \%$ to the values calculated in this way. Fortunately, the $l=2,3$ extrapolations have to be performed only for $R>5.5$ where the post-CCSD(T) contributions are relatively small. Therefore, even if the estimated limits of the T and T(Q) contributions were wrong by $50 \%$, the overall quality of the results would be affected only marginally.

Parenthetically, a typical way to estimate the post-CCSD $(\mathrm{T})$ contributions is to evaluate them is some small basis set and add this value to the final results. As seen from Table 4 , 
this is not a particularly reasonable approach in the present context. In fact, smaller basis sets (i.e. $l=2,3)$ tend to grossly overestimate the post-CCSD(T) effects, sometimes even by a factor of 3 or so. A similar observation has been reported by Smith et al. $\underline{53}$

The final error of the core-core and core-valence contributions to the interaction energy is obtained by summing squares of the errors of all constituents $\left(E_{\text {int,core }}^{\mathrm{CCSD}(\mathrm{T})}, \Delta E_{\text {int,core }}^{\mathrm{T}}, \Delta E_{\text {int,core }}^{(\mathrm{Q})}\right)$ and taking the square root. According to Table 4 this gives the total values of $E_{\text {int(core) }}=$ $53.7 \pm 0.9 \mathrm{~cm}^{-1}$ and $E_{\text {int(core })}=-2.1 \pm 0.5 \mathrm{~cm}^{-1}$ for $R=4.75$ and $R=8.0$, respectively. 
Table 5: Relativistic corrections to the interaction energy of the beryllium dimer. The column "valence" gives results calculated at the valence FCI level of theory and the column "core" provides the inner-shell corrections (see the main text for details). The core correction is neglected for the two-electron Darwin and Breit terms. The interaction energies are given in $\mathrm{cm}^{-1}$ and the internuclear distances in bohr.

\begin{tabular}{|c|c|c|c|c|c|c|c|c|}
\hline \multirow[t]{2}{*}{$l$} & \multicolumn{2}{|c|}{$\left\langle P_{4}\right\rangle$} & \multicolumn{2}{|c|}{$\left\langle D_{1}\right\rangle$} & \multirow{2}{*}{$\begin{array}{c}\text { total } \\
\text { Cowan-Griffin }\end{array}$} & \multirow[t]{2}{*}{$\left\langle D_{2}\right\rangle$} & \multirow[t]{2}{*}{$\langle B\rangle$} & \multirow{2}{*}{$\begin{array}{c}\text { total } \\
\text { Breit-Pauli }\end{array}$} \\
\hline & valence & core & valence & core & & & & \\
\hline & \multicolumn{8}{|c|}{$R=4.75$} \\
\hline 2 & -14.1 & -0.40 & 10.4 & 0.31 & -3.8 & 0.39 & -0.69 & -4.1 \\
\hline 3 & -15.2 & -0.55 & 11.2 & 0.43 & -4.1 & 0.43 & -0.71 & -4.4 \\
\hline 4 & -15.4 & -0.57 & 11.3 & 0.44 & -4.2 & 0.44 & -0.72 & -4.5 \\
\hline$\infty$ & $-15.6 \pm 0.2$ & $-0.60 \pm 0.03$ & $11.5 \pm 0.2$ & $0.47 \pm 0.03$ & $-4.2 \pm 0.3$ & $0.49 \pm 0.05^{\mathrm{a}}$ & $-0.75 \pm 0.03^{\mathrm{a}}$ & $-4.5 \pm 0.3$ \\
\hline 2 & -0.26 & -0.006 & 0.19 & 0.002 & -0.07 & 0.009 & -0.016 & -0.08 \\
\hline 3 & -0.39 & -0.008 & 0.28 & 0.003 & -0.12 & 0.013 & -0.017 & -0.12 \\
\hline 4 & -0.43 & -0.009 & 0.30 & 0.004 & -0.14 & 0.015 & -0.017 & -0.14 \\
\hline$\infty$ & $-0.45 \pm 0.02$ & $-0.01 \pm 0.001$ & $0.32 \pm 0.02$ & $0.005 \pm 0.001$ & $-0.14 \pm 0.03$ & $0.020 \pm 0.005^{\mathrm{a}}$ & $-0.018 \pm 0.001^{\mathrm{a}}$ & $-0.14 \pm 0.03$ \\
\hline
\end{tabular}

athe error estimation includes the uncertainty due to the neglected core contribution 


\subsection{Relativistic corrections}

To meet the high accuracy requirements of this study we must incorporate in our description of the interaction potential the subtle effects of the relativity. As long as the constituting elements are not too heavy, the leading-order relativistic corrections to the molecular energy levels can be calculated by perturbation theory. The approach based on the Breit-Pauli Hamiltonian $\underline{54}$ (accurate to within $\alpha^{2}$ ) is frequently used

$$
\begin{gathered}
E^{(2)}=\left\langle P_{4}\right\rangle+\left\langle D_{1}\right\rangle+\left\langle D_{2}\right\rangle+\langle B\rangle, \\
\left\langle P_{4}\right\rangle=-\frac{\alpha^{2}}{8}\left\langle\sum_{i} \nabla_{i}^{4}\right\rangle, \\
\left\langle D_{1}\right\rangle=\frac{\pi}{2} \alpha^{2} \sum_{a} Z_{a}\left\langle\sum_{i} \delta\left(\mathbf{r}_{i a}\right)\right\rangle, \\
\left\langle D_{2}\right\rangle=\pi \alpha^{2}\left\langle\sum_{i>j} \delta\left(\mathbf{r}_{i j}\right)\right\rangle, \\
\langle B\rangle=\frac{\alpha^{2}}{2}\left\langle\sum_{i>j}\left[\frac{\nabla_{i} \cdot \nabla_{j}}{r_{i j}}+\frac{\mathbf{r}_{i j} \cdot\left(\mathbf{r}_{i j} \cdot \nabla_{j}\right) \nabla_{i}}{r_{i j}^{3}}\right]\right\rangle,
\end{gathered}
$$

where $i$ and $a$ denote electrons and nuclei, respectively, $r_{X Y}$ denotes the interparticle distances, and $\langle\mathcal{O}\rangle$ is the expectation value of an operator $\mathcal{O}$. Further in the paper the above corrections are referred shortly to as the mass-velocity, one-electron Darwin, two-electron Darwin, and orbit-orbit terms (in the order of appearance). Moreover, the sum of $\left\langle P_{4}\right\rangle$ and $\left\langle D_{1}\right\rangle$ terms is called the Cowan-Griffin correction, $\underline{\underline{55}}$ and the names "orbit-orbit" and "Breit" shall be used interchangeably for the term (10). 
In the calculations of the relativistic effects we adopt the following approach. Similarly as for the nonrelativistic energies, the relativistic contributions are divided into the valence and core components. In the case of the two-electron relativistic corrections, $\left\langle D_{2}\right\rangle$ and $\langle B\rangle$, we neglect the core contribution. This is justified because the two-electron contributions are by an order of magnitude smaller than $\left\langle P_{4}\right\rangle$ and $\left\langle D_{1}\right\rangle$ terms, and the core components are further by an order of magnitude smaller than the valence effects. This was verified by carrying out FCI calculations in small basis sets. We estimated that the neglected terms would bring a contribution of only about $0.01 \mathrm{~cm}^{-1}$ to the interaction energy at the minimum of PEC. Thus, they are entirely negligible in the present study, cf. Ref. [23]. Nonetheless, we add an additional uncertainty of $5 \%$ to the calculated two-electron relativistic effects due to the neglected core contributions which is probably a very conservative estimation.

Extrapolations of the relativistic corrections to the complete basis set limit are performed with help of Eq. (3) . The only exception is the two-electron Darwin term where the $l^{-1}$ convergence pattern is found. This is consistent with the numerical experience of Refs. [56, 57] and theoretical findings of Kutzelnigg. .58 In all cases the errors are estimated as the difference between the extrapolated result and the value in the largest basis set.

The valence relativistic corrections are evaluated with the help of the FCI method. The core corrections to the $\left\langle P_{4}\right\rangle$ and $\left\langle D_{1}\right\rangle$ terms were computed at the $\operatorname{CCSD}(\mathrm{T})$ level of theory. In Table 5 we show a short summary of the results for two interatomic distances. One can see that in both cases the relativistic contribution to the interaction energy is non-negligible. Close to the minimum of PEC the relativistic effects decrease the interaction energy by about $5 \mathrm{~cm}^{-1}$ (or $0.5 \%$ ) - a surprisingly large amount for a system as light as the beryllium dimer.

\subsection{Other corrections}

Let us now move to the calculation of the quantum electrodynamics (QED) effects. According to the so-called nonrelativistic QED theory the leading-order post-Breit-Pauli correction to 
Table 6: Quantum electrodynamics contributions to the interaction energy of the beryllium dimer. The core corrections are neglected. The interaction energies are given in $\mathrm{cm}^{-1}$ and the internuclear distances in bohr.

\begin{tabular}{ccccc}
\hline$l$ & $E_{1}^{(3)}$ & $E_{2}^{(3)}$ & $\left\langle H_{A S}\right\rangle$ & total QED \\
\hline \multicolumn{5}{c}{$R=4.75$} \\
\hline 2 & 0.31 & -0.011 & -0.012 & 0.29 \\
3 & 0.34 & -0.012 & -0.013 & 0.32 \\
4 & 0.34 & -0.012 & -0.014 & 0.32 \\
\hline$\infty$ & $0.35 \pm 0.01$ & $-0.014 \pm 0.002$ & $-0.020 \pm 0.007$ & $0.32 \pm 0.02$ \\
\hline \multicolumn{5}{c}{$R=8.00$} \\
\hline 2 & -0.006 & $0.0^{\mathrm{a}}$ & $0.0^{\mathrm{a}}$ & -0.006 \\
3 & -0.008 & $0.0^{\mathrm{a}}$ & $0.0^{\mathrm{a}}$ & -0.008 \\
4 & -0.009 & $0.0^{\mathrm{a}}$ & $0.0^{\mathrm{a}}$ & -0.009 \\
\hline$\infty$ & $-0.009 \pm 0.001$ & $0.0^{\mathrm{a}}$ & $0.0^{\mathrm{a}}$ & $-0.009 \pm 0.001$ \\
\hline
\end{tabular}

abelow $10^{-3} \mathrm{~cm}^{-1}$; impossible to calculate reliably due to large cancellations between the dimer and the monomers

the energy of a molecule in the singlet spin state reads $\underline{49}, \underline{59} \underline{-62}$

$$
E^{(3)}=E_{1}^{(3)}+E_{2}^{(3)}+\left\langle H_{A S}\right\rangle
$$

where $E_{1}^{(3)}$ and $E_{2}^{(3)}$ are the one- and two-electron contributions

$$
\begin{aligned}
& E_{1}^{(3)}=\frac{8 \alpha}{3 \pi}\left(\frac{19}{30}-2 \ln \alpha-\ln k_{0}\right)\left\langle D_{1}\right\rangle \\
& E_{2}^{(3)}=\frac{\alpha}{\pi}\left(\frac{164}{15}+\frac{14}{3} \ln \alpha\right)\left\langle D_{2}\right\rangle
\end{aligned}
$$

and $\left\langle H_{A S}\right\rangle$ is the Araki-Sucher correction ${ }^{63,64}$ given by the formula

$$
\left\langle H_{A S}\right\rangle=-\frac{7 \alpha^{3}}{6 \pi}\left\langle\sum_{i>j} P\left(r_{i j}^{-3}\right)\right\rangle,
$$


where $P\left(r_{i j}^{-3}\right)$ denotes the regularised $r_{i j}^{-3}$ distribution,

$$
\left\langle P\left(r_{i j}^{-3}\right)\right\rangle=\lim _{a \rightarrow 0}\left\langle\theta\left(r_{i j}-a\right) r_{i j}^{-3}+4 \pi\left(\gamma_{E}+\ln a\right) \delta\left(\mathbf{r}_{i j}\right)\right\rangle
$$

and $\gamma_{E} \approx 0.57722 \ldots$ is the Euler-Mascheroni constant. The other new quantity appearing in the above expressions is the Bethe logarithm, $\underline{54,65} \ln k_{0}$.

Let us note that the Araki-Sucher term is formally a two-electron expectation value so it could have been included in $E_{2}^{(3)}$. However, we prefer to consider it separately due to its different nature. Additionally, it may be slightly confusing that the name "one-electron correction" is assigned to $E_{1}^{(3)}$ as $\ln k_{0}$ is a many-electron quantity. However, this establishes a close parallel between the QED and relativistic corrections, cf. Eqs. (66)-(10).

Calculation of the complete leading-order QED corrections for many-electron molecules is notoriously difficult. This is due to the presence of two complicated terms: $\left\langle H_{A S}\right\rangle$ and $\ln k_{0}$. A general method to evaluate the Araki-Sucher correction with the help of the standard quantum chemistry methods has been presented only very recently. $\underline{66}$ This approach has been used in the present paper. Similarly as for the two-electron relativistic corrections we neglect the core contributions to the $\left\langle H_{A S}\right\rangle$ term.

Even more complicated issue is evaluation of the Bethe logarithm, $\ln k_{0}$. Fortunately, for all molecules where the Bethe logarithm is known accurately (hydrogen molecular ion, $\underline{67}-\underline{\underline{70}}$

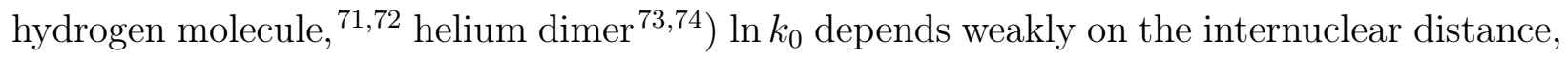
$R$. Therefore, as long as one is not interested in the interaction potential for a very small $R$, the atomic value of $\ln k_{0}$ can be adopted. The Bethe logarithm for the beryllium atom has been evaluated recently by Pachucki and Komasa ${ }^{75}$ and we adopt their value, $\ln k_{0}=5.75034$.

With the help of this approximation $E_{1}^{(3)}$ and $E_{2}^{(3)}$ are obtained by scaling the $\left\langle D_{1}\right\rangle$ and $\left\langle D_{2}\right\rangle$ corrections. The scaling factors do not depend on $R$ and in the present case are approximately equal to 0.0293 and -0.0279 for $E_{1}^{(3)}$ and $E_{2}^{(3)}$, respectively. In Table 6] we present the values of all QED corrections for $R=4.75$ and $R=8.00$. They were 
calculated with the same basis sets as the relativistic effects. The total QED correction is only by an order of magnitude smaller than the Breit-Pauli contribution. This is somewhat contradictory to the estimates based on the order in $\alpha$, but a similar situation is found, e.g. for the hydrogen molecule. $\underline{\underline{71}}$ There are known examples where the QED corrections are even larger than the relativistic ones. $\underline{\underline{76}}, \underline{77}$ Fortunately, such anomalies are absent in the higher-order QED effects. $\underline{78}$

The one-electron term $E_{1}^{(3)}$ dominates the total QED correction and the two-electron effects are smaller by a factor of 20-30. Interestingly, the total QED contribution (12) increases the interaction energy of the beryllium dimer at every point of PEC (i.e. it is attractive). Unfortunately, for larger $R$ we have encountered significant difficulties in calculation of the two-electron QED effects. This is mostly due to the fact that they are very small $\left(<10^{-3} \mathrm{~cm}^{-1}\right)$ and subtraction between the dimer and monomer values leads to a large cancellation of significant digits (cf. Table 6). Therefore, further in the text we neglect the two-electron QED effects and include only the $E_{1}^{(3)}$ term as the dominant contribution to the interaction energy.

We can also estimate the influence of the higher-order relativistic and QED effects on the total interaction energy of the beryllium dimer. Experiences for the helium atom $\frac{77,79}{}$ and the hydrogen molecule ${ }^{78}$ suggest that the dominant term of the $\alpha^{4}$ QED correction is the so-called one-loop diagram $\underline{80}$ given by the following formula

$$
E_{\text {one-loop }}^{(4)}=16 \alpha^{2}\left(\frac{427}{192}-\ln 2\right)\left\langle D_{1}\right\rangle
$$

for a molecule in the singlet electronic state. Since the one-electron Darwin term $\left\langle D_{1}\right\rangle$ has already been calculated in the course of this work, the one-loop term is straightforward to obtain. We find that it contributes as little as about $0.02 \mathrm{~cm}^{-1}$ near the minimum of PEC. Therefore, the higher-order QED effects can safely be neglected within the present accuracy standards and one can rest assured that the QED perturbative series is sufficiently 
well-converged already in the third-order.

Finally, we consider the finite nuclear mass effects. As it is well-known, the leading-order finite nuclear mass correction to the energy of a molecule is the so-called adiabatic correction (also known as the diagonal Born-Oppenheimer correction). As indicated in several previous works, $\underline{22,23}$ this correction is rather small in the present case and we calculate it at the CCSD level of theory. $\frac{81}{}$ For this purpose we employ the Gaussian-type orbitals (GTOs) basis developed by Prascher et al. $\underline{\underline{82}}$ Note that this is the only element of our calculations where we resort to GTOs. Our results indicate that the adiabatic effects are indeed very small for the ground state of the beryllium dimer. For example, they amount only to $-0.14 \mathrm{~cm}^{-1}$ and $-0.02 \mathrm{~cm}^{-1}$ for $R=4.75$ and $R=8.0$, respectively, in line with the simplistic estimates based on the scaling of the $\mathrm{BO}$ interaction energy by the atomic mass. This justifies the neglect of the post-Born-Oppenheimer effects in this study.

The influence of various minor physical effects on the interaction energy of the beryllium dimer is illustrated in Fig. 1, By the term "minor" we mean all non-negligible contributions

Table 7: Optimised parameters of the fit (17) for the non-relativistic Born-Oppenheimer potential energy curve $\left[V^{\mathrm{BO}}(R)\right]$. The symbol $X[ \pm n]$ stands for $X \cdot 10^{ \pm n}$.

\begin{tabular}{cccc}
\hline parameter & $V^{\mathrm{BO}}(R)$ & parameter & $V^{\mathrm{BO}}(R)$ \\
\hline$a$ & $+6.453[-01]$ & $C_{6}$ & $+2.140[+02]^{\mathrm{a}}$ \\
$b$ & $+5.123[-02]$ & $C_{8}$ & $+1.023[+04]^{\mathrm{a}}$ \\
$\eta$ & $+6.052[-01]$ & $C_{10}$ & $+5.165[+05]^{\mathrm{a}}$ \\
\hline$c_{0}$ & $-7.063[+05]$ & $c_{9}$ & $+1.501[+02]$ \\
$c_{1}$ & $+1.647[+06]$ & $c_{10}$ & $-1.359[+01]$ \\
$c_{2}$ & $-1.778[+06]$ & $c_{11}$ & $+9.449[-01]$ \\
$c_{3}$ & $+1.179[+06]$ & $c_{12}$ & $-4.950[-02]$ \\
$c_{4}$ & $-5.376[+05]$ & $c_{13}$ & $+1.888[-03]$ \\
$c_{5}$ & $+1.786[+05]$ & $c_{14}$ & $-4.944[-05]$ \\
$c_{6}$ & $-4.473[+04]$ & $c_{15}$ & $+7.944[-07]$ \\
$c_{7}$ & $-8.612[+03]$ & $c_{16}$ & $-5.899[-09]$ \\
$c_{8}$ & $-1.288[+03]$ & & \\
\hline
\end{tabular}

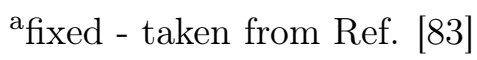




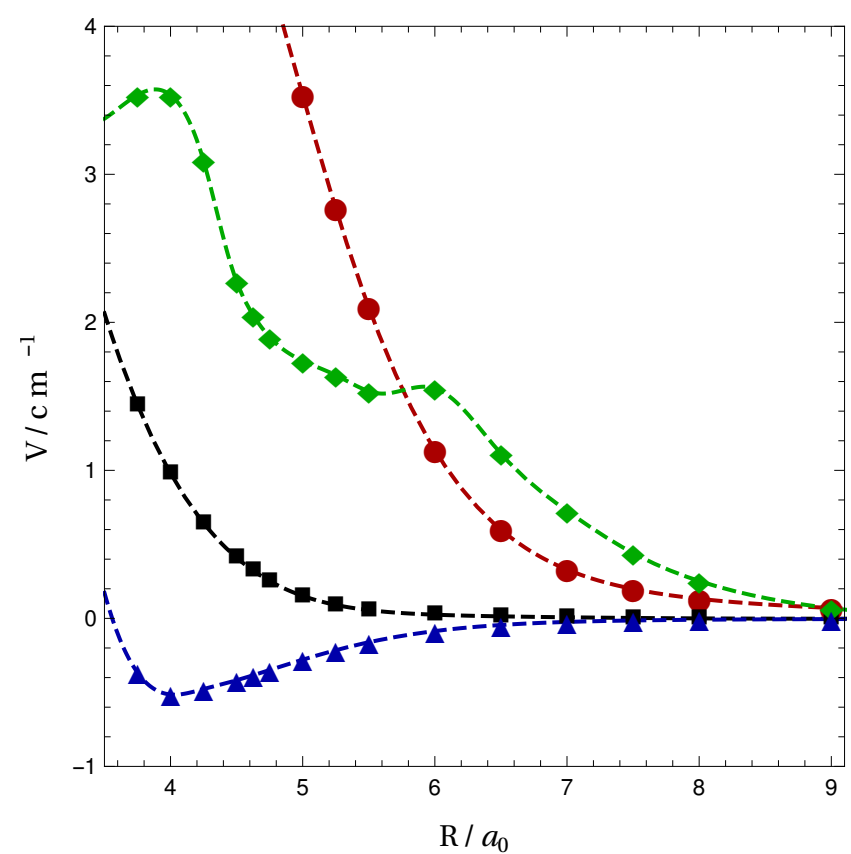

Figure 1: Minor corrections to PEC of the beryllium dimer as a function of the internuclear distance. By red dots, black squares, green diamonds, and blue triangles we denote, respectively, the one-electron relativistic correction, two-electron relativistic correction, post$\operatorname{CCSD}(\mathrm{T})$ inner-shell correction, and one-electron QED correction.

calculated in the course of this work other than the four-electron FCI and the inner-shell $\operatorname{CCSD}(\mathrm{T})$ contributions (which together constitute about $99 \%$ of the total value). Overall, the one-electron relativistic corrections are the most important among the quantities included in Fig. 1, followed by the inner-shell post-CCSD(T) effects. The remaining corrections shown in Fig. 1 are almost by an order of magnitude smaller than the latter two. An interesting feature visible in Fig. 1 is a pronounced hump in the inner-shell post-CCSD(T) corrections curve. We believe that this feature is related to the change in the character of the chemical bond as argued in Ref. [18].

\subsection{Computational details}

Most of the electronic structure calculations described above were carried out with help of the GAMESS program suite. $\stackrel{84}{ }$ The only exceptions are the FCI calculations (performed with HECTOR program ${ }^{50}$ ), higher-order coupled cluster methods (ACESII $\underline{85}$ ) and calculations of 
the adiabatic correction $\left(\mathrm{CFOUR}^{86}\right)$. Matrix elements of the orbit-orbit and Araki-Sucher operators were not evaluated directly in the STOs basis, but with help of the Gaussian fitting technique by using twelve GTOs representing a single exponential orbital. For a single point of the curve $(R=4.75)$ we recomputed all quantities employing fifteen GTOs but the changes were marginal.

To create a complete PEC we selected the following grid of internuclear distances

$$
\begin{aligned}
& \text { from } R=3.75 \text { to } R=5.50 \text { in steps of } 0.25 \text {, } \\
& \text { from } R=5.50 \text { to } R=8.00 \text { in steps of } 0.50 \text {, } \\
& \text { from } R=8.00 \text { to } R=15.0 \text { in steps of } 1.00 \text {, } \\
& \text { from } R=15.0 \text { to } R=25.0 \text { in steps of } 2.50 \text {, }
\end{aligned}
$$

in atomic units. Additionally, we evaluated a single point at $R=4.625$ to improve the description of the minimum of PEC. This gives a total number of 25 points with increasing spacings, so that the grid is more dense in regions with larger variations of the total interaction energy.

\section{Analytic fits of the potentials}

The raw ab initio data points were fitted with the conventional analytic form frequently used for the atom-atom interactions

$$
V(R)=e^{-a R-b R^{2}} \sum_{k=0}^{N_{p}} c_{k} R^{k}-\sum_{n=3}^{N_{a}} f_{2 n}(\eta R) \frac{C_{2 n}}{R^{2 n}}
$$

where $f_{2 n}(\eta R)$ are the Tang-Toennies damping functions. $\underline{.77}$ This expression contains three nonlinear parameters $(a, b$, and $\eta)$ and an adjustable number of the linear parameters $\left(N_{p}\right)$ and the asymptotic constants $\left(N_{a}\right)$. In the case of the BO potential we employ the asymptotic constants $C_{2 n}$ evaluated with more accurate theoretical methods. For the $C_{6}, C_{8}$, and $C_{10}$ 
Table 8: Optimised parameters of the fit (17) for the one-electron relativistic corrections, see Eqs. (7) and (8). The symbol $X[ \pm n]$ stands for $X \cdot 10^{ \pm n}$.

\begin{tabular}{ccc}
\hline parameter & $V^{\mathrm{P} 4}(R)$ & $V^{\mathrm{D} 1}(R)$ \\
\hline$a$ & $+1.180[+00]$ & $+1.128[+00]$ \\
$b$ & $+1.099[-01]$ & $+3.231[-02]$ \\
$\eta$ & $+9.410[-01]$ & $+1.083[+00]$ \\
\hline$c_{0}$ & $-1.759[+00]$ & $-1.556[-01]$ \\
$c_{1}$ & $+9.444[-01]$ & $+6.433[-02]$ \\
$c_{2}$ & $-1.298[-01]$ & $-5.854[-03]$ \\
\hline$C_{6}$ & $+4.106[-01]$ & $-2.717[-01]$ \\
$C_{8}$ & $+6.406[+01]$ & $-4.371[+01]$ \\
$C_{10}$ & $-2.908[+03]$ & $+1.786[+03]$ \\
\hline
\end{tabular}

dispersion coefficients we adopt the values reported by Porsev and Derevianko $\stackrel{83,88}{2}$ which are in a very good agreement with the earlier results of Mitroy and Bromley. ${ }^{89}$ No reliable data is available for the higher-order constants so they are neglected here.

Unfortunately, the BO results for the beryllium dimer are very difficult to fit with a smooth analytic function. This is clearly related to the unusual shape of this curve illustrated in Fig. 2. We needed as many as 16 parameters to obtain an accurate fit of the BO potential. The optimised values are given in Table 7. Further work is necessary to reduce the number of parameters.

The generic formula (17) is also used for the fitting of the relativistic corrections. Each correction defined by Eq. (6) is fitted separately. Unfortunately, we are not aware of any reliable asymptotic constants which could be used for the present purposes. Therefore, we use Eq. (17) with $N_{p}=2$ and $N_{a}=3$ and obtain approximate dispersion coefficients directly from the fit. This leaves nine free parameters to be determined by the fitting procedure which is sufficient to obtain a satisfactory accuracy. The only exception from the procedure described above is found for the orbit-orbit correction, Eq. (10), which possesses the $C_{4} / R^{4}$ long-range asymptotics. .90 Therefore, instead of the $C_{6}-C_{10}$ coefficients we use leading-order $C_{4}-C_{8}$ constants as free parameters. The one-electron QED correction is obtained by scaling 
Table 9: Optimised parameters of the fit (17) for the two-electron relativistic corrections, see Eqs. (9) and (10) for the definitions. The symbol $X[ \pm n]$ stands for $X \cdot 10^{ \pm n}$.

\begin{tabular}{ccc}
\hline parameter & $V^{\mathrm{D} 2}(R)$ & $V^{\mathrm{B}}(R)$ \\
\hline$a$ & $+4.278[-01]$ & $-7.024[-02]$ \\
$b$ & $+7.388[-02]$ & $+9.394[-02]$ \\
$\eta$ & $+1.128[+00]$ & $+8.485[+00]$ \\
\hline$c_{0}$ & $-5.700[-04]$ & $-9.031[-05]$ \\
$c_{1}$ & $+2.373[-04]$ & $+2.196[-05]$ \\
$c_{2}$ & $-2.343[-05]$ & $-1.519[-06]$ \\
\hline$C_{4}$ & - & $+1.839[-04]$ \\
$C_{6}$ & $-1.582[-02]$ & $-3.636[-03]$ \\
$C_{8}$ & $-7.303[-01]$ & $-3.762[-02]$ \\
$C_{10}$ & $-2.015[+02]$ & - \\
\hline
\end{tabular}

of the $\left\langle D_{1}\right\rangle$ relativistic correction according to the formula (12). The fitting errors are by an order of magnitude smaller than the estimated uncertainties of the respective theoretical results. The final optimised values of the fitting parameters are given in Table 8 and Table 9 and the complete PEC is illustrated in Fig. 2. The raw ab initio data used for fitting are included in the supplementary material of this paper.

\section{Spectroscopic data}

The total PEC generated in this work was used to calculate the spectroscopic parameters of the ground state of the beryllium dimer. The well-depth $\left(D_{e}\right)$ and the equilibrium bond length $\left(R_{e}\right)$ are obtained by finding the minimum of the PEC numerically. This gives the values of $D_{e}=934.5 \mathrm{~cm}^{-1}$ and $R_{e}=2.4425 \AA$. We estimate that the error of the theoretically determined well-depth is at most $2.5 \mathrm{~cm}^{-1}$.

Let us now compare these results with the experimental and theoretical data available in the literature. The original experimental result of Merritt et al. $\frac{36}{\text { is }} D_{e}=929.7 \mathrm{~cm}^{-1}$ employing the expanded Morse oscillator (EMO) model of the potential. This choice is less 


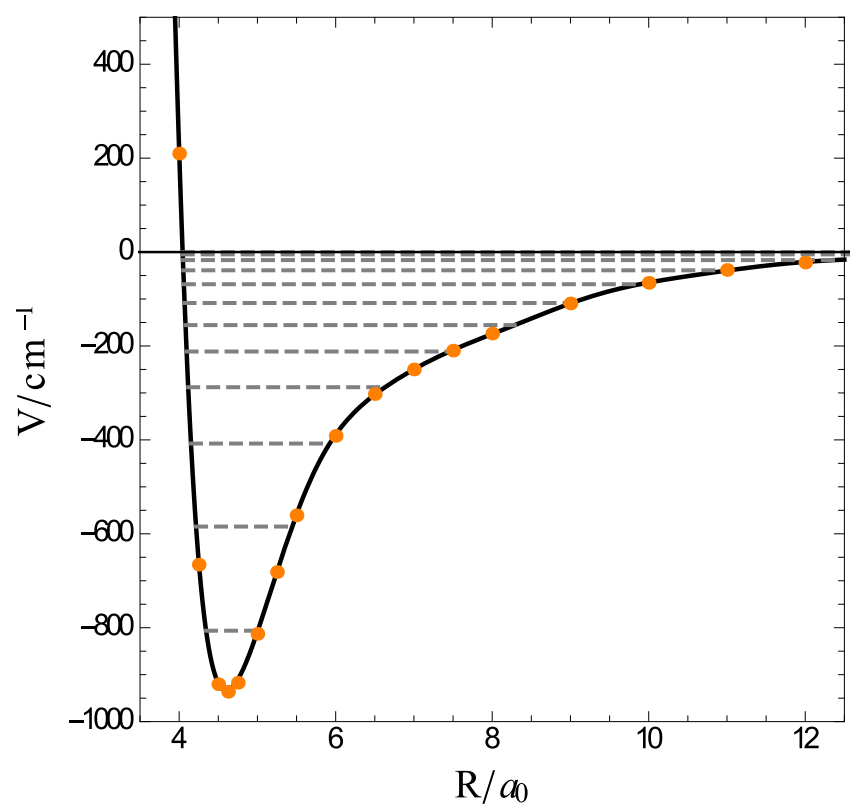

Figure 2: Complete PEC for the $X^{1} \Sigma_{g}^{+}$state of $\mathrm{Be}_{2}$ (solid black line); orange dots are the extrapolated ab initio data points. The horizontal dashed lines are energies of the $J=0$ vibrational levels. The horizontal black solid line denotes the onset of the continuum.

than ideal for the beryllium dimer due to an unphysical decay at large interatomic distances. This deficiency was first pointed out by Patkowski et al. $\underline{\underline{38}}$ who employed "morphed" ab initio potential energy curves with the correct asymptotics. Depending on the number of parameters used in the morphing procedure the value of $D_{e}$ varied in the range $933.0-934.6 \mathrm{~cm}^{-1}$, in almost perfect agreement with the present result. A similarly good agreement is found with the recent work of Meshkov et al. $\underline{\underline{32}}$ where two empirical potentials have been determined by the direct-potential-fit procedure. The Morse-long range (MLR) and Chebyshev polynomial expansion (CPE) functions give $D_{e}=934.8 \pm 0.3 \mathrm{~cm}^{-1}$ and $D_{e}=935.0 \pm 0.3 \mathrm{~cm}^{-1}$, respectively. The most recent ab initio result of Koput, $\underline{\underline{22}} D_{e}=935 \pm 10 \mathrm{~cm}^{-1}$, is also well within the present error bars. In Table 10 we show a compilation of the spectroscopic data obtained from selected experimental measurements, semi-empirical/morphed potentials, and pure $a b$ initio calculations.

Let us note that in our recent theoretical work ${ }^{23}$ we have predicted the well-depth to be $D_{e}=929.0 \pm 1.9 \mathrm{~cm}^{-1}$. This is outside the error bars of the present work and vice versa. Both results have been obtained with a very similar method, so this discrepancy requires 
a more detailed explanation. This difference can mostly be attributed to the fact that the internuclear distance adopted in Ref. [23] does not correspond to the true minimum of the theoretical PEC. In Ref. [23] the calculations were performed only for a single $R$ from the work of Merritt et al. $\underline{36}\left(R_{e}=2.4536 \AA\right)$. This value differs from the minimum of the potential energy curve determined in the present work by more than $0.01 \AA$. To resolve the discrepancy we repeated the calculations of Ref. [23] using exactly the same methodology (basis sets, extrapolations, electronic structure methods, etc.) but with the value of $R$ found here. We obtained $D_{e}=931.7 \mathrm{~cm}^{-1}$ which is significantly closer to the results of the present work. A slightly increased uncertainty of the present results comes mostly from the inaccuracy of the fit. We recommend that the present result $\left(D_{e}=934.5 \pm 2.5 \mathrm{~cm}^{-1}\right)$ is referenced in other works instead of the value given in Ref. [23].

Table 10: Comparison of the selected empirical and theoretical results for the ground state of the beryllium dimer. The energies are given in $\mathrm{cm}^{-1}$ and the equilibrium distances in Angström, $\AA$. The symbol $E_{b}(\nu=11)$ denotes the binding energy of the last (twelfth) vibrational level and the remaining abbreviations are defined in the main text.

\begin{tabular}{cccccc}
\hline Ref. & method & $D_{e}$ & $D_{0}$ & $R_{e}$ & $E_{b}(\nu=11)$ \\
\hline \multicolumn{6}{c}{ empirical/morphed potentials } \\
\hline$[34]$ & $\nu$ extrapolation & $790 \pm 30$ & 660 & 2.45 & - \\
{$[36]$} & EMO & $929.7 \pm 2.0$ & 806.53 & 2.4536 & - \\
{$[31]$} & morphed 3-param. & 922.9 & 795.0 & 2.4382 & - \\
{$[38]$} & morphed 5-param. & 934.6 & 807.4 & 2.438 & 0.42 \\
{$[32]$} & MLR potential fit & $934.8 \pm 0.3$ & 808.16 & 2.445 & 0.518 \\
{$[32]$} & CPE potential fit & $935.0 \pm 0.3$ & 808.20 & 2.445 & 0.521 \\
\hline \multicolumn{6}{c}{ pure ab initio potentials } \\
\hline$[19]$ & CCSD(T)+FCI & $944 \pm 25$ & 816 & 2.440 & - \\
{$[24]$} & CAS $r_{12}$-MR-ACPF & $898 \pm 8$ & 772 & 2.444 & - \\
{$[20]$} & EXRHF & $945 \pm 15$ & 819 & 2.452 & - \\
{$[21]$} & CCSD $(\mathrm{T})+$ FCI & $938 \pm 15$ & - & 2.44 & - \\
{$[22]$} & CCSD $(\mathrm{T})+$ FCI & $935 \pm 10$ & 808.3 & 2.444 & 0.4 \\
this work & see the text & $934.5 \pm 2.5$ & 808.0 & 2.4425 & 0.51 \\
\hline
\end{tabular}

Finally, we solve the (radial) nuclear Schrödinger equation with the help of the DVR method $\frac{91}{}$ to obtain the vibrational energy levels. The results are listed in Table 11 and compared with the experimental results of Merritt et al. $\underline{36}$ We find a very good agreement 
between the theoretical and empirical vibrational energy terms. The average deviation is only about $1 \mathrm{~cm}^{-1}$ indicating that the spectroscopic accuracy has indeed been achieved. Additionally, we note that the experimental uncertainty of the data of Ref. [36] is about 0.5 $\mathrm{cm}^{-1}$, so that the accuracy of our ab initio results might be slightly better than the average deviation suggests. Crucially, our PEC supports twelve vibrational energy levels confirming the prediction of Patkowski et al. $\stackrel{38}{\underline{3}}$ The last vibrational level calculated with the current PEC lies just about $0.5 \mathrm{~cm}^{-1}$ below the onset of the continuum. This is in a good agreement both with Ref. [38] where a value in the range $0.40-0.44 \mathrm{~cm}^{-1}$ was predicted, and with the more recent Ref. [32] where the value of $0.52 \mathrm{~cm}^{-1}$ was obtained. Despite our results favour the latter value, the accuracy of PEC developed in this work is not sufficient to give a definite answer.

Table 11: Comparison of the vibrational spectra $E(\nu)-E(\nu=0)$ for the $X^{1} \Sigma_{g}^{+}$state of the beryllium dimer. The experimental values from Ref. $\underline{\underline{36}}$ are listed in the second column, the $a b$ initio values obtained in this work are listed in the third column, and the deviations between the latter two are given in the last column. All values are given in $\mathrm{cm}^{-1}$.

\begin{tabular}{cccc}
\hline$\nu$ & \multicolumn{3}{c}{$E(\nu)-E(\nu=0)$} \\
\hline & exp. & this work & deviation \\
\hline 1 & 222.6 & 223.5 & 0.9 \\
2 & 397.1 & 398.9 & 1.8 \\
3 & 518.1 & 520.5 & 2.4 \\
4 & 594.8 & 596.6 & 1.8 \\
5 & 651.5 & 652.9 & 1.4 \\
6 & 698.8 & 699.9 & 1.1 \\
7 & 737.7 & 738.5 & 0.8 \\
8 & 768.2 & 769.1 & 0.9 \\
9 & 789.9 & 790.5 & 0.6 \\
10 & 802.6 & 803.0 & 0.4 \\
11 & - & 807.5 & - \\
\hline$\delta_{r m s}$ & - & - & 1.3 \\
\hline
\end{tabular}




\section{Conclusions}

The present work reports a detailed first-principles theoretical study of the ground electronic state of the beryllium dimer. An accurate $a b$ initio potential energy curve for this system has been calculated with a composite scheme employing several quantum-chemical methods and large basis sets composed of Slater-type orbitals. The dominant (four-electron) valence contribution to the interaction energy has been calculated at the FCI level of theory. The remaining inner-shell effects are treated with high-level coupled cluster methods such as $\operatorname{CCSD}(\mathrm{T})$ or $\operatorname{CCSDT}(\mathrm{Q})$.

To further increase the accuracy of our theoretical predictions we have calculated corrections due to some small physical effects. These include the relativistic corrections (full Breit-Pauli Hamiltonian) and the leading-order QED corrections. The finite nuclear mass effects (the non-Born-Oppenheimer effects) are found to be negligible at present.

Spectroscopic parameters generated from the PEC developed in this work show a remarkably good agreement with the experimental data. This is true for the well-depth (calculated $\left.D_{e}=934.5 \pm 2.5 \mathrm{~cm}^{-1}\right)$, dissociation energy $\left(D_{0}=808.0 \mathrm{~cm}^{-1}\right)$, and the equilibrium bond length $\left(R_{e}=2.4425 \AA\right)$. The vibrational energy terms are on the average only $1 \mathrm{~cm}^{-1}$ away from the empirical results of Merritt et al. $\underline{\underline{36}}$ showing that the spectroscopic accuracy has been achieved. We have confirmed the existence of the last (twelfth) vibrational state and predicted that it lies just $0.5 \mathrm{~cm}^{-1}$ below the onset of the continuum. Lastly, this study has proven that the Slater-type orbitals can routinely be used as a basis set for quantum-chemical calculations for diatomic systems.

\section{Acknowledgement}

The authors thank Bogumił Jeziorski for reading and commenting on the manuscript. M.L. acknowledges the financial support from the Polish National Science Centre under Grant No. 2016/21/N/ST4/03732. R.M. and J.G.B. were supported by the Polish National Science 
Centre through Grant No. 2016/21/B/ST4/03877. Computations presented in this research were carried out with the support of the Interdisciplinary Centre for Mathematical and Computational Modelling (ICM) at the University of Warsaw, grant number G59-29.

\section{Supporting Information Available}

The following files are available free of charge.

The following files are available free of charge.

- supp.pdf: contains raw ab initio data used for fitting and detailed compositions of the STOs basis sets.

\section{References}

(1) Fraga, S.; Ransil, B. J. Studies in Molecular Structure. VII. Limited Configuration Interaction for Selected First-Row Diatomics. J. Chem. Phys. 1962, 36, 1127-1142.

(2) Bender, C. F.; Davidson, E. R. Theoretical Calculation of the Potential Curves of the $\mathrm{Be}_{2}$ Molecule. J. Chem. Phys. 1967, 47, 4972-4978.

(3) Dykstra, C. E.; Schaefer, H. F.; Meyer, W. Electron correlation in small metal clusters. Application of a theory of self-consistent electron pairs to the $\mathrm{Be}_{4}$ system. J. Chem. Phys. 1976, 65, 5141-5146.

(4) Blomberg, M. R. A.; Siegbahn, P. E. M. Beryllium dimer, a critical test case of MBPT and CI methods. Int. J. Quantum Chem. 1978, 14, 583-592.

(5) Chiles, R. A.; Dykstra, C. E. An electron pair operator approach to coupled cluster wave functions. Application to $\mathrm{He}_{2}, \mathrm{Be}_{2}$, and $\mathrm{Mg}_{2}$ and comparison with CEPA methods. J. Chem. Phys. 1981, 74, 4544-4556. 
(6) Liu, B.; McLean, A. D. Ab initio potential curve for $\mathrm{Be}_{2}$ from the interacting correlated fragments method. J. Chem. Phys. 1980, 72, 3418-3419.

(7) Harrison, R.; Handy, N. Full CI results for $\mathrm{Be}_{2}$ and $\left(\mathrm{H}_{2}\right)_{2}$ in large basis sets. Chem. Phys. Lett. 1983, 98, $97-101$.

(8) Mukherjee, D.; Moitra, R. K.; Mukhopadhyay, A. Applications of a non-perturbative many-body formalism to general open-shell atomic and molecular problems: calculation of the ground and the lowest $\pi-\pi^{*}$ singlet and triplet energies and the first ionization potential of trans-butadiene. Mol. Phys. 1977, 33, 955-969.

(9) Jeziorski, B.; Monkhorst, H. J. Coupled-cluster method for multideterminantal reference states. Phys. Rev. A 1981, 24, 1668-1681.

(10) Mahapatra, U. S.; Datta, B.; Mukherjee, D. A state-specific multi-reference coupled cluster formalism with molecular applications. Mol. Phys. 1998, 94, 157-171.

(11) Bauschlicher, C. W.; Langhoff, S. R.; Partridge, H. Theoretical study of the BeLi, BeNa, MgLi, MgNa, and AlBe molecules and their negative ions. J. Chem. Phys. 1992, 96, $1240-1247$.

(12) Füsti-Molnár, L.; Szalay, P. G. High-quality theoretical potential energy surface for $\mathrm{Be}_{2}$ by using the multireference averaged quadratic coupled-cluster (MR-AQCC) method and large basis sets. Chem. Phys. Lett. 1996, 258, $400-408$.

(13) Füsti-Molnár, L.; Szalay, P. G. New Versions of Approximately Extensive Corrected Multireference Configuration Interaction Methods. J. Phys. Chem. 1996, 100, 62886297.

(14) Stärck, J.; Meyer, W. The ground state potential of the beryllium dimer. Chem. Phys. Lett. 1996, 258, $421-426$. 
(15) Kaledin, L.; Kaledin, A.; Heaven, M.; Bondybey, V. Electronic structure of $\mathrm{Be}_{2}$ : theoretical and experimental results. Dedicated to Professor Keiji Morokuma in celebration of his 65th birthday. J. Mol. Struct.: Theochem 1999, 461-462, $177-186$.

(16) Schmidt, M. W.; Ivanic, J.; Ruedenberg, K. Electronic Structure Analysis of the Ground-State Potential Energy Curve of $\mathrm{Be}_{2}$. J. Phys. Chem. A 2010, 114, 8687-8696.

(17) Mitin, A. V. Ab initio calculations of weakly bonded $\mathrm{He}_{2}$ and $\mathrm{Be}_{2}$ molecules by MRCI method with pseudo-natural molecular orbitals. Int. J. Quantum Chem. 2010, 111, $2560-2567$.

(18) El Khatib, M.; Bendazzoli, G. L.; Evangelisti, S.; Helal, W.; Leininger, T.; Tenti, L.; Angeli, C. Beryllium Dimer: A Bond Based on Non-Dynamical Correlation. J. Phys. Chem. A 2014, 118, 6664-6673.

(19) Martin, J. M. The ground-state spectroscopic constants of $\mathrm{Be}_{2}$ revisited. Chem. Phys. Lett. 1999, 303, $399-407$.

(20) Røeggen, I.; Veseth, L. Interatomic potential for the $\mathrm{X}^{1} \Sigma$ state of $\mathrm{Be}_{2}$, revisited. Int. J. Quantum Chem. 2004, 101, 201-210.

(21) Patkowski, K.; Podeszwa, R.; Szalewicz, K. Interactions in Diatomic Dimers Involving Closed-Shell Metals. J. Phys. Chem. A 2007, 111, 12822-12838.

(22) Koput, J. The ground-state potential energy function of a beryllium dimer determined using the single-reference coupled-cluster approach. Phys. Chem. Chem. Phys. 2011, 13, 20311-20317.

(23) Lesiuk, M.; Przybytek, M.; Musiał, M.; Jeziorski, B.; Moszynski, R. Reexamination of the calculation of two-center, two-electron integrals over Slater-type orbitals. III. Case study of the beryllium dimer. Phys. Rev. A 2015, 91, 012510. 
(24) Gdanitz, R. J. Accurately solving the electronic Schrodinger equation of atoms and molecules using explicitly correlated (r12-)MR-CI.: The ground state of beryllium dimer $\left(\mathrm{Be}_{2}\right)$. Chem. Phys. Lett. 1999, 312, $578-584$.

(25) Pecul, M.; Jaszuński, M.; Larsen, H.; Jørgensen, P. Singlet excited states of Be $2 . J$. Chem. Phys. 2000, 112, 3671-3679.

(26) Sharma, S.; Yanai, T.; Booth, G. H.; Umrigar, C. J.; Chan, G. K.-L. Spectroscopic accuracy directly from quantum chemistry: Application to ground and excited states of beryllium dimer. J. Chem. Phys. 2014, 140, 104112.

(27) Blunt, N. S.; Smart, S. D.; Kersten, J. A. F.; Spencer, J. S.; Booth, G. H.; Alavi, A. Semi-stochastic full configuration interaction quantum Monte Carlo: Developments and application. J. Chem. Phys. 2015, 142, 184107.

(28) Deible, M. J.; Kessler, M.; Gasperich, K. E.; Jordan, K. D. Quantum Monte Carlo calculation of the binding energy of the beryllium dimer. J. Chem. Phys. 2015, 143, 084116.

(29) Magoulas, I.; Bauman, N. P.; Shen, J.; Piecuch, P. Application of the CC(P;Q) Hierarchy of Coupled-Cluster Methods to the Beryllium Dimer. J. Phys. Chem. A 2018, 122, 1350-1368.

(30) Karton, A.; McKemmish, L. K. Can Popular DFT Approximations and Truncated Coupled Cluster Theory Describe the Potential Energy Surface of the Beryllium Dimer? Aust. J. Chem. 2018,

(31) Špirko, V. Potential energy curve of $\mathrm{Be}_{2}$ in its ground electronic state. J. Mol. Spectr. 2006, 235, $268-270$.

(32) Meshkov, V. V.; Stolyarov, A. V.; Heaven, M. C.; Haugen, C.; LeRoy, R. J. Direct- 
potential-fit analyses yield improved empirical potentials for the ground $\mathrm{X}^{1} \Sigma_{g}^{+}$state of $\mathrm{Be}_{2}$. J. Chem. Phys. 2014, 140, 064315.

(33) Bondybey, V. E.; English, J. H. Laser vaporization of beryllium: Gas phase spectrum and molecular potential of $\mathrm{Be}_{2}$. J. Chem. Phys. 1984, 80, 568-570.

(34) Bondybey, V. Electronic structure and bonding of $\mathrm{Be}_{2}$. Chem. Phys. Lett. 1984, 109, $436-441$.

(35) Bondbey, V. E. Laser-Induced Fluorescence and Bonding of Metal Dimers. Science 1985, 227, 125-131.

(36) Merritt, J. M.; Bondybey, V. E.; Heaven, M. C. Beryllium Dimer-Caught in the Act of Bonding. Science 2009, 324, 1548-1551.

(37) Bernath, P. F. Extracting Potentials from Spectra. Science 2009, 324, 1526-1527.

(38) Patkowski, K.; Špirko, V.; Szalewicz, K. On the Elusive Twelfth Vibrational State of Beryllium Dimer. Science 2009, 326, 1382-1384.

(39) Slater, J. C. Atomic Shielding Constants. Phys. Rev. 1930, 36, 57-64.

(40) Slater, J. C. Analytic Atomic Wave Functions. Phys. Rev. 1932, 42, 33-43.

(41) Lesiuk, M.; Moszynski, R. Reexamination of the calculation of two-center, two-electron integrals over Slater-type orbitals. I. Coulomb and hybrid integrals. Phys. Rev. E 2014, 90, 063318.

(42) Lesiuk, M.; Moszynski, R. Reexamination of the calculation of two-center, two-electron integrals over Slater-type orbitals. II. Neumann expansion of the exchange integrals. Phys. Rev. E 2014, 90, 063319.

(43) Lesiuk, M. Calculation of STOs electron repulsion integrals by ellipsoidal expansion and large-order approximations. J. Math. Chem. 2016, 54, 572-591. 
(44) Mohr, P. J.; Newell, D. B.; Taylor, B. N. CODATA recommended values of the fundamental physical constants: 2014. Rev. Mod. Phys. 2016, 88, 035009.

(45) Meija, J.; Coplen, T. B.; Berglund, M.; Brand, W. A.; Bièvre, P. D.; Gröning, M.; Holden, N.; Irrgeher, J.; Loss, R.; Walczyk, T.; Prohaska, T. Atomic weights of the elements 2013 (IUPAC Technical Report). Pure Appl. Chem. 2016, 88, 265-291.

(46) Lesiuk, M.; Tucholska, A. M.; Moszynski, R. Combining Slater-type orbitals and effective core potentials. Phys. Rev. A 2017, 95, 052504.

(47) Dunning, T. H. Gaussian basis sets for use in correlated molecular calculations. I. The atoms boron through neon and hydrogen. J. Chem. Phys. 1989, 90, 1007-1023.

(48) Hill, R. N. Rates of convergence and error estimation formulas for the Rayleigh-Ritz variational method. J. Chem. Phys. 1985, 83, 1173-1196.

(49) Pachucki, K.; Komasa, J. Relativistic and QED Corrections for the Beryllium Atom. Phys. Rev. Lett. 2004, 92, 213001.

(50) Przybytek, M. FCI program HeCTOR. 2014,

(51) Boys, S.; Bernardi, F. The calculation of small molecular interactions by the differences of separate total energies. Some procedures with reduced errors. Mol. Phys. 1970, 19, $553-566$.

(52) Raghavachari, K.; Trucks, G. W.; Pople, J. A.; Head-Gordon, M. A fifth-order perturbation comparison of electron correlation theories. Chem. Phys. Lett. 1989, 157, $479-$ 483.

(53) Smith, D. G. A.; Jankowski, P.; Slawik, M.; Witek, H. A.; Patkowski, K. Basis Set Convergence of the Post-CCSD(T) Contribution to Noncovalent Interaction Energies. J. Chem. Theory Comput. 2014, 10, 3140-3150. 
(54) H. A. Bethe, E. E. S. Quantum Mechanics of One- and Two- Electron Systems; Springer: Berlin, 1975.

(55) Cowan, R. D.; Griffin, D. C. Approximate relativistic corrections to atomic radial wave functions. J. Opt. Soc. Am. 1976, 66, 1010-1014.

(56) Ottschofski, E.; Kutzelnigg, W. Direct perturbation theory of relativistic effects for explicitly correlated wave functions: The He isoelectronic series. J. Chem. Phys. 1997, $106,6634-6646$.

(57) Halkier, A.; Helgaker, T.; Klopper, W.; Olsen, J. Basis-set convergence of the twoelectron Darwin term. Chem. Phys. Lett. 2000, 319, 287 - 295.

(58) Kutzelnigg, W. Relativistic corrections to the partial wave expansion of two-electron atoms. Int. J. Quantum Chem. 2008, 108, 2280-2290.

(59) Caswell, W.; Lepage, G. Effective lagrangians for bound state problems in QED, QCD, and other field theories. Phys. Lett. B 1986, 167, $437-442$.

(60) Pachucki, K. Higher-order binding corrections to the Lamb shift. Ann. Phys. 1993, 226, 1-87.

(61) Pachucki, K. Simple derivation of helium Lamb shift. J. Phys. B 1998, 31, 5123.

(62) Pachucki, K. Higher-order effective Hamiltonian for light atomic systems. Phys. Rev. A 2005, 71, 012503.

(63) Araki, H. Quantum-Electrodynamical Corrections to Energy-Levels of Helium. Progr. Theor. Phys. 1957, 17, 619-642.

(64) Sucher, J. Energy Levels of the Two-Electron Atom to Order $\alpha^{3}$ Ry; Ionization Energy of Helium. Phys. Rev. 1958, 109, 1010-1011.

(65) Schwartz, C. Lamb Shift in the Helium Atom. Phys. Rev. 1961, 123, 1700-1705. 
(66) Balcerzak, J. G.; Lesiuk, M.; Moszynski, R. Calculation of Araki-Sucher correction for many-electron systems. Phys. Rev. A 2017, 96, 052510.

(67) Bukowski, R.; Jeziorski, B.; Moszynski, R.; Kołos, W. Bethe logarithm and Lamb shift for the hydrogen molecular ion. Int. J. Quantum Chem. 1992, 42, 287-319.

(68) Korobov, V. I. Bethe logarithm for the hydrogen molecular ion $\mathrm{HD}^{+}$. Phys. Rev. A 2004, 70, 012505.

(69) Korobov, V. I. Bethe logarithm for the hydrogen molecular ion $\mathrm{H}_{2}{ }^{+}$. Phys. Rev. A 2006, 73, 024502.

(70) Korobov, V. I.; Hilico, L.; Karr, J.-P. Calculation of the relativistic Bethe logarithm in the two-center problem. Phys. Rev. A 2013, 87, 062506.

(71) Piszczatowski, K.; Łach, G.; Przybytek, M.; Komasa, J.; Pachucki, K.; Jeziorski, B. Theoretical Determination of the Dissociation Energy of Molecular Hydrogen. J. Chem. Theory Comput. 2009, 5, 3039-3048.

(72) Komasa, J.; Piszczatowski, K.; Łach, G.; Przybytek, M.; Jeziorski, B.; Pachucki, K. Quantum Electrodynamics Effects in Rovibrational Spectra of Molecular Hydrogen. J. Chem. Theory Comput. 2011, 7, 3105-3115.

(73) Przybytek, M.; Cencek, W.; Komasa, J.; Each, G.; Jeziorski, B.; Szalewicz, K. Relativistic and Quantum Electrodynamics Effects in the Helium Pair Potential. Phys. Rev. Lett. 2010, 104, 183003.

(74) Cencek, W.; Przybytek, M.; Komasa, J.; Mehl, J. B.; Jeziorski, B.; Szalewicz, K. Effects of adiabatic, relativistic, and quantum electrodynamics interactions on the pair potential and thermophysical properties of helium. J. Chem. Phys. 2012, 136, 224303.

(75) Puchalski, M.; Komasa, J.; Pachucki, K. Testing quantum electrodynamics in the lowest singlet states of the beryllium atom. Phys. Rev. A 2013, 8\%, 030502. 
(76) Puchalski, M.; Piszczatowski, K.; Komasa, J.; Jeziorski, B.; Szalewicz, K. Theoretical determination of the polarizability dispersion and the refractive index of helium. Phys. Rev. A 2016, 93, 032515.

(77) Pachucki, K. $\alpha^{4} \mathcal{R}$ corrections to singlet states of helium. Phys. Rev. A 2006, 74, 022512.

(78) Puchalski, M.; Komasa, J.; Czachorowski, P.; Pachucki, K. Complete $\alpha^{6} m$ Corrections to the Ground State of $\mathrm{H}_{2}$. Phys. Rev. Lett. 2016, 117, 263002.

(79) Pachucki, K. Helium energy levels including $m \alpha^{6}$ corrections. Phys. Rev. A 2006, 74, 062510.

(80) Eides, M. I.; Grotch, H.; Shelyuto, V. A. Theory of light hydrogenlike atoms. Phys. Rep. 2001, 342, $63-261$.

(81) Gauss, J.; Tajti, A.; Kállay, M.; Stanton, J. F.; Szalay, P. G. Analytic calculation of the diagonal Born-Oppenheimer correction within configuration-interaction and coupledcluster theory. J. Chem. Phys. 2006, 125, 144111.

(82) Prascher, B. P.; Woon, D. E.; Peterson, K. A.; Dunning, T. H.; Wilson, A. K. Gaussian basis sets for use in correlated molecular calculations. VII. Valence, core-valence, and scalar relativistic basis sets for $\mathrm{Li}, \mathrm{Be}, \mathrm{Na}$, and $\mathrm{Mg}$. Theor. Chem. Acc. 2011, 128, 69-82.

(83) Porsev, S. G.; Derevianko, A. High-accuracy calculations of dipole, quadrupole, and octupole electric dynamic polarizabilities and van der Waals coefficients $C_{6}, C_{8}$, and $C_{10}$ for alkaline-earth dimers. J. Exp. Theor. Phys. 2006, 102, 195-205.

(84) Schmidt, M. W.; Baldridge, K. K.; Boatz, J. A.; Elbert, S. T.; Gordon, M. S.; Jensen, J. H.; Koseki, S.; Matsunaga, N.; Nguyen, K. A.; Su, S.; Windus, T. L.; Dupuis, M.; Montgomery, J. A. General atomic and molecular electronic structure system. J. Comp. Chem. 1993, 14, 1347-1363. 
(85) Stanton, J. F.; Gauss, J.; Watts, J. D.; Lauderdale, W. J.; Bartlett, R. J. AcesII Program System Release 2.0 QTP; University of Florida: Gainesville, FL, 1994.

(86) Stanton, J. F.; Gauss, J.; Harding, M. E.; Szalay, P. G.; et al., CFour: a program package for performing high-level quantum chemical calculations on atoms and molecules, see http://www.cfour.de.

(87) Tang, K. T.; Toennies, J. P. An improved simple model for the van der Waals potential based on universal damping functions for the dispersion coefficients. J. Chem. Phys. 1984, 80, 3726-3741.

(88) Porsev, S. G.; Derevianko, A. High-accuracy relativistic many-body calculations of van der Waals coefficients $C_{6}$ for alkaline-earth-metal atoms. Phys. Rev. A 2002, 65, 020701.

(89) Mitroy, J.; Bromley, M. W. J. Semiempirical calculation of van der Waals coefficients for alkali-metal and alkaline-earth-metal atoms. Phys. Rev. A 2003, 68, 052714.

(90) Meath, W. J.; Hirschfelder, J. O. Long-Range (Retarded) Intermolecular Forces. J. Chem. Phys. 1966, 44, 3210-3215.

(91) Colbert, D. T.; Miller, W. H. A novel discrete variable representation for quantum mechanical reactive scattering via the S-matrix Kohn method. J. Chem. Phys. 1992, 96, 1982-1991. 